\title{
Using the Own Flexibility of a Climbing Robot as a Double Force Sensor
}

\author{
J.A.Somolinos ${ }^{1, a}$, R. Morales ${ }^{2, b}$, C. Morón ${ }^{3, c}$ and A. García ${ }^{3, d}$ \\ ${ }^{1}$ ETS Ingenieros Navales Univ. Politécnica de Madrid. Arco de la Victoria sn 28040 Madrid, SPAIN \\ ${ }^{2}$ ETS Ing. Industriales Univ. Castilla-La Mancha. Campus Universitario sn 02071 Albacete, SPAIN \\ ${ }^{3}$ E.U. Arquitectura. Universidad Politécnica de Madrid. Juan de Herrera s/n 28040 Madrid, SPAIN \\ joseandres.Somolinos@upm.es, ${ }^{b}$ rafael.morales@uclm.es , ${ }^{c}$ carlos.moron@upm.es, \\ dalfonso.garciag@upm.es.
}

Keywords: Force sensor, Flexible Robot, Climbing Robot, Mechanisms.

\begin{abstract}
Force sensors are used when interaction tasks are carried out by robots in general, and by climbing robots in particular. If the mechanics and electronics systems are contained inside the own robot, the robot becomes portable without external control. Commercial force sensors cannot be used due to limited space and weight. By selecting the links material with appropriate stiffness and placing strain gauges on the structure, the own robot flexibility can be used such as force sensor. Thus, forces applied on the robot tip can be measured without additional external devices. Only gauges and small internal electronic converters are necessary. This paper illustrates the proposed algorithm to achieve these measurements. Additionally, experimental results are presented.
\end{abstract}

\section{Introduction}

Force sensors have been used in industrial robotics from 1980's. Commercial versions (See [1] as example), based on the Maltese-cross force sensor [5], consist on a cylindrical device with elastic elements inside where 8 (or 8 pairs) strain gauges are mounted. The sensor location is between the wrist plate and the tool. In general, these devices can measure both, forces and torques, with respect to the wrist reference frame. These measurements let the programmers carry out different force or hybrid position-force control tasks while interacting with its environment. Some typical industrial applications are: polishing, machining or assembly [6].

MATS-ASIBOT is a robot manipulator of 5 DOF (Degrees Of Freedom) which weight is about $10 \mathrm{~kg}$, that can reach $1.37 \mathrm{~m}$ and with a payload of $2 \mathrm{~kg}$ over each of its two tips. Its main feature is the fact that all the control system is on-board and then, it only needs an external power supply (See [2]). The robot has a symmetrical structure which allows it to climb between static and simple docking stations situated into the environment. Additionally, the robot is equipped with special conical connectors at every tip. It allows the robot to perform different movements, as well the possibility to change tools for special tasks or applications.

The robot needs two force sensors for both tips. One of the sensors is placed between the wrists and the tool, and the other is placed between the docking station and the wrists. To maintain all the robot properties and, at the same time, to include onboard the additional elements that conform the force sensors, it is necessary a new design of the robot. Thus, this method is a non-viable option. This paper shows how forces can be achieved from both ends of the robot. The method uses deflections measurements of the own robot structure [3,4]. These measurements are obtained from strain gauges placed over the two main links and they are disposed in such a way that all forces which produce deflections are measured. Thus, the robot acts as a flexible structure, and there is no other necessity of inserting external flexible elements. After a brief description of the robot stiffness model, experimental setup and results are presented. 


\section{Compliance and Stiffness modeling.}

A simple two bars mechanism with linear elasticity model is used for modeling the flexibility of the MATS-ASIBOT. Fig. 1 illustrates the proposed scheme including forces, torques, linear and angular deflections over the robot structure.

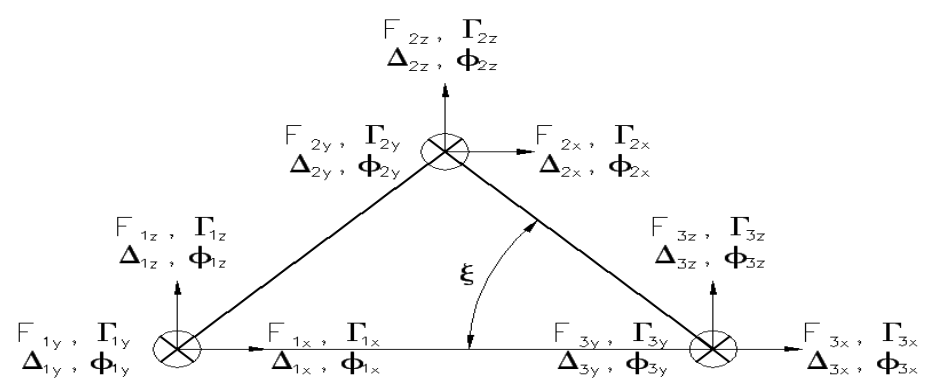

Figure 1: Generalized forces and deflections over the structure with two links and three nodes

To obtain the relations between forces and deflections, two matrices are defined:

$$
\left(\begin{array}{l}
\mathbf{W}_{\mathrm{k}} \\
\mathbf{W}_{\mathrm{u}}
\end{array}\right)^{(12 \times 1+6 \times 1)}=\left(\begin{array}{lllllll}
\mathbf{F}_{2} & \Gamma_{2} & \mathbf{F}_{3} & \Gamma_{3} & \mathbf{F}_{1} & \Gamma_{1}
\end{array}\right)^{\top},\left(\begin{array}{c}
\Delta_{\mathrm{u}} \\
\boldsymbol{\Delta}_{\mathrm{k}}
\end{array}\right)^{(12 \times 1+6 \times 1)}=\left(\begin{array}{lllllll}
\Delta_{2} & \boldsymbol{\Phi}_{2} & \Delta_{3} & \boldsymbol{\Phi}_{3} & \Delta_{1} & \boldsymbol{\Phi}_{1}
\end{array}\right)^{\top}
$$

Where $\mathbf{F}_{\mathrm{i}}=\left(\begin{array}{lll}\mathrm{F}_{\mathrm{ix}} & \mathrm{F}_{1 \mathrm{y}} & \mathrm{F}_{1 \mathrm{z}}\end{array}\right)$ and $\boldsymbol{\Gamma}_{\mathrm{i}}=\left(\begin{array}{lll}\boldsymbol{\Gamma}_{\mathrm{ix}} & \boldsymbol{\Gamma}_{\mathrm{iy}} & \boldsymbol{\Gamma}_{\mathrm{iz}}\end{array}\right)$ denote forces and torques, while the linear and angular deflections over node 'i' are denoted as $\Delta_{\mathrm{i}}=\left(\begin{array}{lll}\Delta_{\mathrm{ix}} & \Delta_{\mathrm{iy}} & \Delta_{\mathrm{iz}}\end{array}\right)$ and $\boldsymbol{\Phi}_{\mathrm{i}}=\left(\begin{array}{lll}\phi_{\mathrm{ix}} & \phi_{\mathrm{iy}} & \phi_{\mathrm{iz}}\end{array}\right)$. W and $\Delta$ denote generalized forces and deflections respectively. Sub indices $\mathrm{u}$ and $\mathrm{k}$ indicate unknown and known parameters respectively. The known values of vector $\mathbf{W}, \mathbf{W}_{\mathrm{k}}$, are the corresponding null values over node 2 and the values to be measured, while the known values of $\Delta, \Delta_{k}$, are the displacements of the robot base, which are considered null. Although, base and tip of the robot are interchangeable nodes, node 1 is considered a fixed one and node 3 represents the mobile tip.

Relation between tip forces and tip deflections is obtained. Compliance coefficients are angular joints dependant, and they can be expressed as a function of the "configuration angle" $\xi$. If more complex structures are considered, numerical computation is required, but in our case, due to the symmetry of the structure, coefficients are obtained in a symbolic way. If only tip forces and tip deflections are taken into account, the whole compliance matrix is not necessary and only a 3x3 sub matrix has to be computed. Then, the compliance matrix $-\xi$ dependant- becomes (details from [7]):

$$
\begin{aligned}
& \Delta_{\text {tip }}=\mathbf{C}(\xi) \cdot \mathbf{F}_{\text {tip }} \text { or } \quad\left(\begin{array}{c}
\Delta_{\text {tip }} \text { x } \\
\Delta_{\text {tip }} \mathrm{y} \\
\Delta_{\text {tip }} \mathrm{z}
\end{array}\right)=\left(\begin{array}{ccc}
\mathrm{C}_{11}(\xi) & 0 & \mathrm{C}_{13}(\xi) \\
0 & \mathrm{C}_{22}(\xi) & 0 \\
\mathrm{C}_{13}(\xi) & 0 & \mathrm{C}_{33}(\xi)
\end{array}\right) \cdot\left(\begin{array}{l}
\mathrm{F}_{\text {tip }} \mathrm{x} \\
\mathrm{F}_{\text {tip }} \mathrm{y} \\
\mathrm{F}_{\text {tip }} \mathrm{z}
\end{array}\right) \\
& \mathrm{C}_{11}(\xi)=\frac{\mathrm{L}^{3}}{3} \cdot\left(\frac{1}{\mathrm{E}_{1} \mathrm{I}_{\mathrm{y} 1}}+\frac{1}{\mathrm{E}_{2} \mathrm{I}_{\mathrm{y} 2}}\right) \cdot \sin ^{2}(\xi) \\
& \mathrm{C}_{13}(\xi)=\frac{\mathrm{L}^{3}}{3} \cdot\left(\frac{2}{\mathrm{E}_{1} \mathrm{I}_{\mathrm{y} 1}}+\frac{1}{\mathrm{E}_{2} \mathrm{I}_{\mathrm{y} 2}}\right) \cdot \sin (\xi) \cdot \cos (\xi) \\
& \mathrm{C}_{22}(\xi)=\frac{\mathrm{L}_{3}}{3} \cdot\left[\left(\frac{1}{\mathrm{E}_{2} \mathrm{I}_{z 2}}+\frac{7}{\mathrm{E}_{1} \mathrm{I}_{\mathrm{z} 1}}\right) \cos ^{4}(\xi)+\left(\frac{2}{\mathrm{E}_{2} \mathrm{I}_{\mathrm{z} 2}}+\frac{12}{\mathrm{G}_{1} \mathrm{I}_{\mathrm{x} 1}}-\frac{4}{\mathrm{E}_{1} \mathrm{I}_{\mathrm{z} 1}}\right) \sin ^{2}(\xi) \cos ^{2}(\xi)+\left(\frac{1}{\mathrm{E}_{2} \mathrm{I}_{\mathrm{z} 2}}+\frac{1}{\mathrm{E}_{1} \mathrm{I}_{\mathrm{z} 1}}\right) \sin ^{4}(\xi)\right] \\
& \mathrm{C}_{33}(\xi)=\frac{\mathrm{L}^{3}}{3} \cdot\left(\frac{7}{\mathrm{E}_{1} \mathrm{I}_{\mathrm{y} 1}}+\frac{1}{\mathrm{E}_{2} \mathrm{I}_{\mathrm{y} 2}}\right) \cdot \cos ^{2}(\xi)
\end{aligned}
$$

Being $\mathbf{C}(\xi)$ a symmetric and positive definite matrix. The stiffness matrix $\mathbf{K}(\xi)$ is obtained by algebraic inversion. Frequencies of the vibration modes depend on the stiffness and inertia matrices $[3,4]$, and they are $\xi$ dependants. The selection of the flexible materials was carried out avoiding the excitation of the high vibration frequencies at the expected highest robot speed. 


\section{Experimental setup}

A general view of MATS-ASIBOT is showed in Fig. 2 and 3. Fig. 2 depicts the robot, disposed in a vertical way, while Fig. 3 illustrates the robot interacting with an element of its environment. As it can be observed, four docking stations have been placed on a metallic support station.

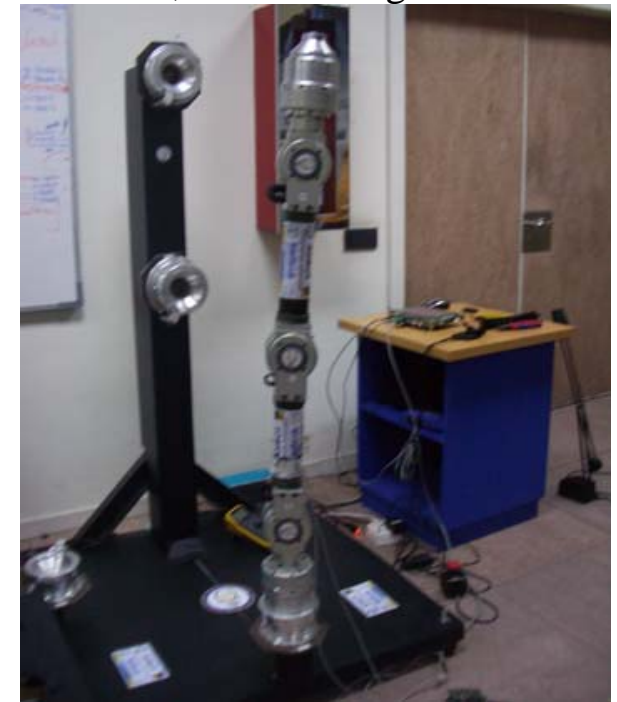

Figure 2: Initial and final position of the robot

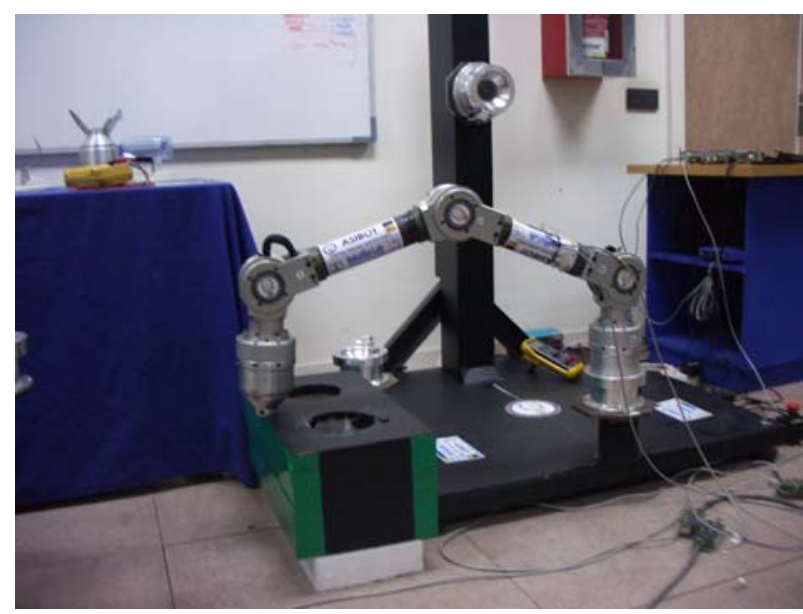

Figure 3. Applying a force over an object

From the mechanical structure point of view, two main beams with finite stiffness are linked with the three main robot joints, which are considered rigid. From five strain gauges bridges placed on every flexible robot link and their conditioners, voltage signals, are obtained from AD converters. Fig. 4 shows a modeling scheme of the measurement system. From ADC measurements, and by real-time computation of the robot gains and stiffness matrices, tip forces are obtained.

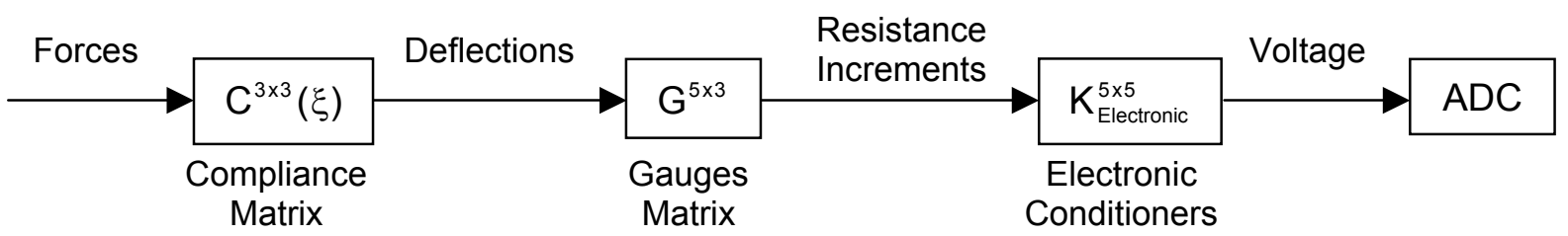

Figure 4: Schematic modeling of the sensorial system

\section{Experimental Results}

Fig. 5 depicts a graphical representation of the robot. The robot is considered both, rigid and flexible (with exagerated deflections), when the robot moves in a free way and only gravity forces are applied on it. Fig. 6 shows the geometric form of the robot when it interacts with an object and reaction forces are applied on the tip. Both set of forces have been reproduced experimentally by performing a trajectory, which is described below.

The trajectory has been decomposed into five time segments (See Fig. 7): During segment 1 and 5, the robot is stopped at a vertical posture (as in Fig. 2) and no flexion is produced. During segment 3 , the robot interacts with the object (Fig. 3 and 6) and positive flexion of the structure let us to measure positive values of forces. In segments 2 and 4, the robot moves in a free way (Fig. 5), and negative flexion indicates negative forces applied which corresponds with gravity effects. Fig. 7 shows the expected force from this trajectory and the force measured by the proposed method and the five time segments are clearly ploted. The excellent matching between theoretical and experimental data justifes the goodness of the proposed method. 


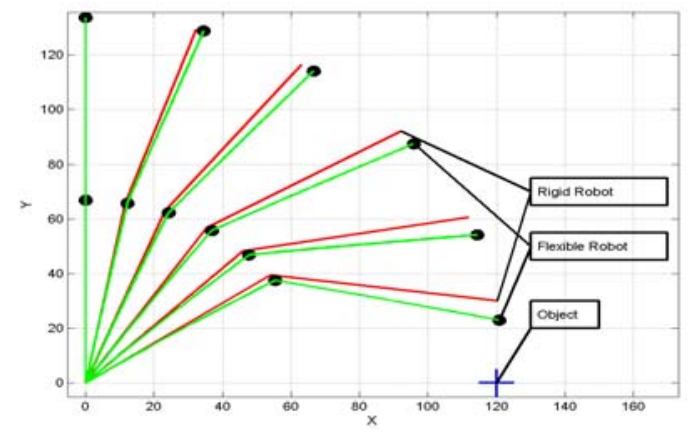

Figure 5. Geometric computation of the Rigid/Flexible robot during its free motion

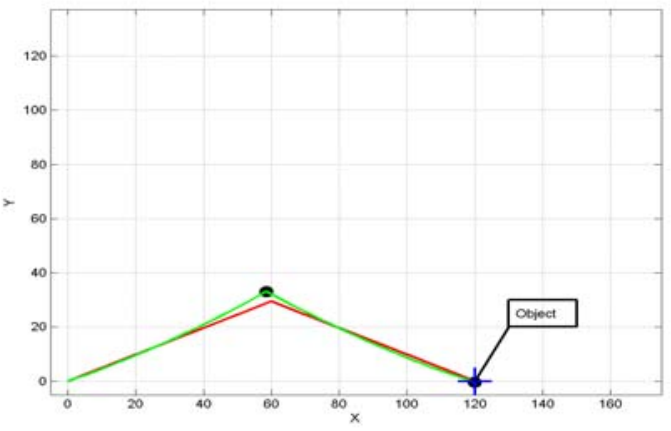

Figure 6. Geometric computation of the Rigid/Flexible robot when object interaction.

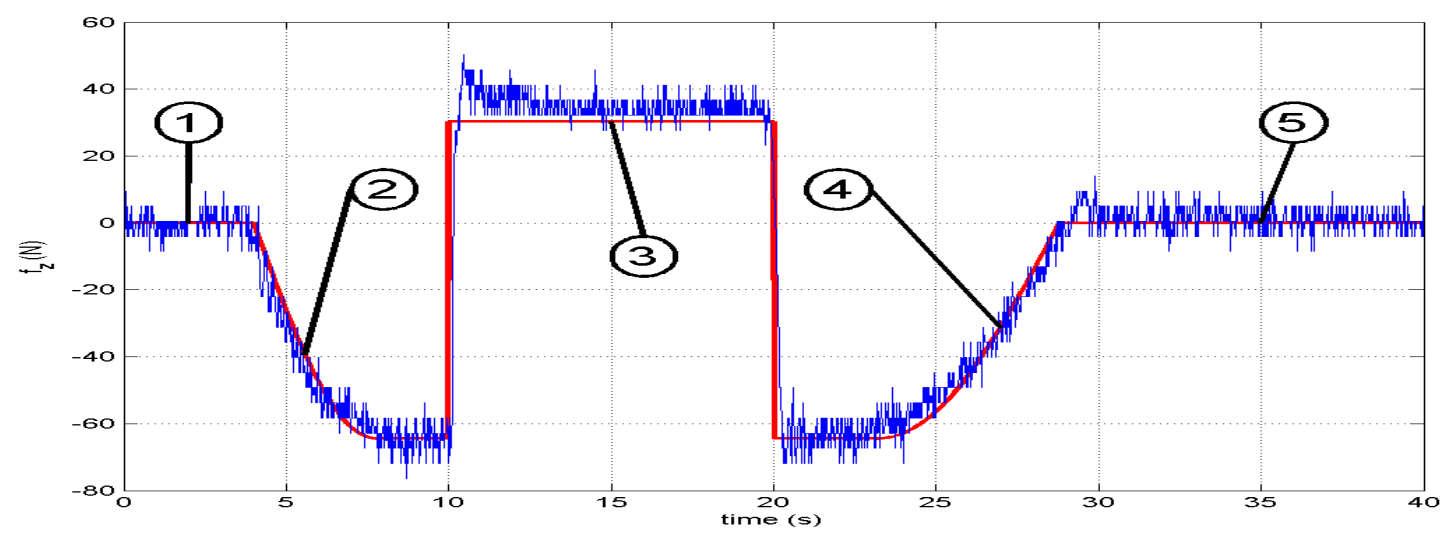

Figure 7. Measured and estimated force ( $\mathrm{N}$ vs seconds) during the complete robot motion

\section{Conclusions}

The use of two sets of strain gauges on the robot links captures the flexion/torsion deflectionss of the robot structure and it makes the system capable to measure efforts. The strain gauges were placed symmetrically on the structure. The effort measurements were achieved without any interlayer element without the wrist and the tool and between the wrist and the docking station where the robot is anchored. The proposed system is considered as a double force sensor in the sense that both sets of strain gauges let to measure all the forces acting on the robot, including efforts that result from the weight of the robot itself. Experimental results justify the effectiveness of the proposed method. By the addition of the signal conditioners, easily placed into the robot, the use of large and weighted commercial force sensors is avoided.

\section{Acknowledgements:}

Prof. Somolinos wishes to thank the faculty and staff of the Robotics Lab at the University Carlos III of Madrid their excellent reception during his stay which allowed the realization of this work.

\section{References}

[1] Ati Industrial Automation. Multi-Axis Force/Torque Sensor. www.ati-ia.com

[2] C.Balaguer; A.Giménez; A.Jardón. "The MATS robot: Service Climbing Robot for Personal Assistance”. IEEE Robotics \& Automation Magazine. Vol. 13. N. 1. pp.51-58. 2006.

[3] Feliu, V., García A., Somolinos J.A., "Experimental Testing of a Gauge Based Collision Detection Mechanism for a new 3DOF Flexible Robot”, Journal Robotics Systems. Vol.20, N.6 pp. 271-284 2003

[4] Feliu, V., García A., Somolinos J.A., "Gauge Based Tip Position Control of a new Three-degree-offreedom Flexible Robot”, Journal of Robotics Research. Vol. 20 N8 pp.660-675, 2001

[5] V.D.Scheinman, "Design of a computer controlled manipulator", Ph.D. Thesis. Stanford University.1969

[6] L. Sciaviccco and B Siciliano "Modelling and Control of Robot Manipulators" Springer Verlag. 2000.

[7] J.A.Somolinos, V. Feliu and L. Sánchez "Design, Dynamic Modeling and Experimental Validation of a New Three-Degree-Of-Freedom Flexible Arm”. Mechatronics. Vol. 12 (7) pp. 919-948. 2002 